\title{
JMSR
}

Journal of Medical

and Scientific Research

\section{Evaluation of pre-analytical phase performance of emergency laboratory by harmonized quality indicators and six sigma}

\author{
Veerendra Kumar Arumalla ${ }^{1, *}$, Selvakumar Chelliah $^{2}$ and Madhubala $\mathbf{V}^{1}$ \\ ${ }^{1}$ Department of Biochemistry, ESIC Medical College \& PGIMSR, KK Nagar, Chennai-600078, Tamil Nadu, India. \\ ${ }^{2}$ Department of Biochemistry, Employees State Insurance \&Post graduate Institute of Medical Education and Research, Manicktala \\ Kolkata-700054, West Bengal, India.
}

\begin{abstract}
Background: Pre-analytical errors account for up to $70 \%$ of all the errors made in laboratory diagnostics which are mostly not directly under laboratory control. Laboratories across the world have been using different Quality indicators (QIs) for identifying and quantification of pre-analytical errors. Objective of the present study is to identify the different pre-analytical errors with their frequency and to assess the pre-analytical phase performance of emergency laboratory by using harmonized Quality Indicators and six sigma metrics.
\end{abstract}

Methods and material: A prospective observational study was conducted from January 2019 to December 2019 to monitor the inappropriateness of samples and test request forms. We have quantified the performance of pre-analytical phase of our emergency laboratory based on the harmonized QIs proposed by The International Federation of Clinical Chemistry Working Group on Laboratory Errors and Patient Safety (IFCC- WGLEPS) and six sigma metrics.

Results: Emergency laboratory received a total of 55431 samples during Jan- 2019 to Dec- 2019. Number of pre-analytical errors were 1089 which accounted for $1.96 \%$ of total samples received. Haemolysed samples, clotted samples and samples with insufficient volume were contributed to $37 \%, 26 \%$ and $15 \%$ of the total preanalytical errors respectively.

Conclusions: Pre-analytical phase performance of our emergency laboratory complies with the quality specifications laid by the International Federation of Clinical Chemistry Working Group on Laboratory Errors and Patient Safety (IFCC-WGLEPS). Implementation of harmonised QIs assures the comparability of laboratory findings with different laboratories across the world.

Keywords: pre-analytical errors; harmonized quality indicators; IFCCWG-LEPS

*Corresponding author: Veerendra Kumar Arumalla, Associate Professor, Department of Biochemistry, ESIC Medical College \& PGIMSR, KK Nagar, Chennai-600078, Tamil Nadu, India. Mobile: +91 8072959429; Email: drveerendraarumalla@gmail.com

Received 12 December 2020; Revised 20 February 2021; Accepted 26 February 2021; Published 10 March 2021

Citation: Arumalla VK, Chelliah S, Madhubala V. Evaluation of pre-analytical phase performance of emergency laboratory by harmonized quality indicators and six sigma. J Med Sci Res. 2021; 9(2):64-70. DOI: http://dx.doi.org/10.17727/JMSR.2021/9-10

Copyright: (C) 2021 Arumalla VK et al. Published by KIMS Foundation and Research Center. This is an open-access article distributed under the terms of the Creative Commons Attribution License, which permits unrestricted use, distribution, and reproduction in any medium, provided the original author and source are credited. 


\section{Introduction}

Quality in laboratory medicine should be defined as the guarantee that each and every step in the total testing process (TTP) is correctly performed, thus assuring valuable medical decision-making and effective patient care [1]. Laboratory total testing process involves pre-analytical, analytical and postanalytical phases. Pre-analytical phase starts from test request to the delivery of the specimen to the laboratory [2, 3].

Pre-analytical errors account for up to $70 \%$ of all the errors made in laboratory diagnostics. Most of these are related to, patient preparation, patient identification, sample collection, transportation and storage [4]. These pre-analytical phase procedures of testing process are performed outside the laboratory and are not under direct control of the laboratory.

Pre-analytical errors largely interfere with the test analysis thus affects patient treatment. These errors are proven to be a burden for the laboratory and a serious issue for the hospital administration as sample rejection can leads to loss of critical time and adds to the cost of patient care $[5,6]$.

Most of the Quality Indicators (QIs) in laboratory medicine tend to focus on the performance and efficiency of analytical processes with less preference to pre-analytical and post-analytical phases [7]. But existing evidence suggests that most errors in the loop actually fall outside the analytical phase, and the pre- and post-analytical steps have been found to be more vulnerable to the risk of error [8]. The lack of attention to extra laboratory factors is the main reason for the multitude of errors that continue to occur in the pre-analytical phase.

Requirements for accreditation against ISO 15189:2012 (Medical laboratories - Requirements for quality and competence) emphasizes that there is a need to evaluate, monitor and improve all the procedures of pre-analytical phase including test requesting, patient and sample identification, sample collection, sample handling, transportation and storage [9].

Identification and effective use of quality indicators of pre-analytical phase is therefore an essential requirement for laboratory accreditation and for useful risk management strategy. In recent years different QIs have been used in different laboratories in order to comply with the requirement of accreditation standards. Due to non-uniformity of methods used by different laboratories for the identification and management of QIs, the results obtained cannot be compared [1].

Standardization in this area of identification and effective use of Quality indicators of pre- analytical phase underpins the effective reporting of errors. This will enable the comparisons between peers and identification of areas for improvements in the TTP. These QIs then provide a harmonized platform for targeted continuous improvement and a means of measuring said improvements [1]. The International Federation of Clinical Chemistry Working Group on Laboratory Errors and Patient Safety (IFCCWGLEPS) has worked to improve awareness in the field of laboratory errors and patient safety, developed pilot studies to monitor error rates and implemented projects to reduce errors [10, 11]. IFCC-WGLEPS has developed harmonized quality Indicators (QIs) to assess the total testing process of the clinical laboratory (Pre-analytical, analytical and post-analytical). They have also proposed Quality Specifications (QS) for each QI.

A clear definition of how QIs are evaluated and the development of performance levels (e.g., unacceptable, minimum, and desirable) are useful for laboratory performance evaluation, especially in the pre-analytical phase.

Six sigma methodology is another method of quality assessment, which is applicable in the pre-analytical phase. Six sigma provides principles and tools that can be applied when the outcome of the process can be measured. The number of errors or defects done by the laboratory can be quantified using six sigma metrics. Bill Smith, known colloquially as the father of Six Sigma, decided to measure the defects per million (DPM) instead of defects per thousand. The number of errors, or DPM, is a measure of laboratory performance [12].

In order to measure the quality of pre-analytical phase on six sigma scale, it requires monitoring of outcome process, counting the defects and calculating DPM. By using the statistical tables DPMs can be converted to sigma metrics [13]. Quality is assessed on a sigma scale, from 3 sigma as the minimum allowed for 
routine performance to six sigma as best-in-class quality. World-class quality processes have a six sigma level, which means around 3.4 errors per million [12].

Most of the existing studies reported pre-analytical errors by using different QIs which are not harmonized and makes them difficult to compare the results across the different laboratories. On the contrary the present study used harmonized QIs to assess and report the pre-analytical errors.

Objective of the current study is to quantify different pre-analytical errors and to assess the performance of pre-analytical phase of emergency laboratory based on the harmonized QIs proposed by IFCCWGLEPS and Six sigma metrics.

\section{Materials and methods}

A prospective observational study was conducted in Emergency Laboratory, Department of Biochemistry, from January 2019 to December 2019 to monitor the inappropriateness of samples and test request forms (TRFs). We have quantified the performance of preanalytical phase of our emergency laboratory based on the harmonized QIs proposed by IFCC- WGLEPS and Six sigma metrics.

Laboratory setting: Emergency laboratory is equipped with fully automated Chemistry analysers: Roche Cobas C311, Vitro 350, ABG analysers: Radiometer ABL 80 Flex, Cobas b123, Coagulation analyser: ACL Elite Pro, Fiske Osmometer: Osmolality serum \&Urine, Alere Triage meterPro: cardiac markers, Cobas h 232: D-dimer, Beckman Access 2 for analysing Immunoassay parameters.

Path of samples: Samples collected from different ICUs, Casualty and wards are transported to emergency laboratory by nursing staff. Once the samples reaches the laboratory technician will check the TRFs which will be signed and stamped by the clinicians and registers the sample in LIS (SUKRAA), barcode with SID( sample identity number)will be generated for each sample.

Followed by registration in LIS, samples are processed for analysis. After centrifugation Unsuitable samples and inappropriate test requests were recognised and identified by virtual inspection by laboratory staff. The laboratory staff working in emergency laboratory are trained to identify and register all the errors that may affect the total testing process, including those that occur in the pre-analytical phase. All unsuitable samples and inappropriate test requests (as per QIs in the table 1) will be notified in LIS and informed immediately to the concerned department.

\section{Data collection}

Harmonized QIs proposed by IFCC-WGLEPS [11] were used to quantify the performance of the emergency laboratory in pre-analytical phase.

The following QIs were used to quantify the errors concerning patient identification, errors concerning physician identification were evaluated by QI 5 \& 6 respectively. Errors concerning test input- QI 7, lost-not received Q I8, Inappropriate container QI 9, Haemolysed samples QI10, Clotted samples QI 11, Insufficient sample volume QI 12, Inadequate sample-anticoagulant volume ratio QI 13 and Improperly labelled samples QI 15 (Table 1).

During the study period we have counted the QIs on monthly basis. Descriptions for each QI were mentioned in the Table 1. To assess our performance of pre-analytical phase of our emergency laboratory we have compared our results with the quality specifications (QS) reported by IFCC- WGLEPS [11].

\section{Statistical analysis}

Pre-analytical errors were calculated for each month and distribution of inappropriateness among the different ICUs and wards were calculated using Microsoft Excel.

We calculated the sigma metric for QIs. First, we calculated the DPM rate using the following formula:

$\mathrm{DPM}=($ number of errors $\times 1,000,000) /$ total number of specimens or requests.

The DPM rate was converted to a sigma value based on tables available online https://www. westgard.com/sixsigtable.htm. For example, for the QI involving hemolyzed samples, we calculated the sigma value as follows:

DPM $=$ (number of hemolyzed samples biochemistry $\times 1,000,000) /$ total number of samples [12]. 
Table 1: Quality indicators in the pre-analytic phase proposed by IFCC- WGLEPS [1, 11].

\begin{tabular}{|c|c|}
\hline QI code & Description \\
\hline QI 1: Appropriateness of test request & Number of requests without clinical question/ Total number of requests \\
\hline QI 2: Appropriateness of test request & $\begin{array}{l}\text { Number of inappropriate requests, with respect to clinical question / Number of } \\
\text { requests reporting clinical } \\
\text { Question }\end{array}$ \\
\hline QI 3: Examination requisition & Number of requests without physician identification / Total number of requests \\
\hline QI 4: Examination requisition & Number of unintelligible requests / Total number of requests \\
\hline QI 5: Identification & $\begin{array}{l}\text { Number of requests with errors concerning patient identification / Total number of } \\
\text { requests }\end{array}$ \\
\hline QI 6: Identification & $\begin{array}{l}\text { Number of requests with errors concerning physician identification / Total number of } \\
\text { requests }\end{array}$ \\
\hline QI 7: Test request & Number of requests with errors concerning test input / Total number of requests \\
\hline QI 8: Samples & Number of samples lost-not received / Total number of samples \\
\hline QI 9: Samples & Number of samples collected in inappropriate container / Total number of samples \\
\hline QI 10: Samples & Number of haemolysed samples / Total number of samples \\
\hline QI 11: Samples & Number of clotted samples / Total number of samples with anticoagulant \\
\hline QI 12: Samples & Number of samples with insufficient sample volume / Total number of samples \\
\hline QI 13: Samples & $\begin{array}{l}\text { Number of samples with inadequate sample-anticoagulant volume ratio / Total number } \\
\text { of samples with anticoagulant }\end{array}$ \\
\hline QI 14: Samples & Number of damaged samples / Total number of samples \\
\hline QI 15: Samples & Number of improperly labelled samples / Total number of samples \\
\hline QI 16: Samples & Number of improperly stored samples / Total number of samples \\
\hline
\end{tabular}

\section{Results}

Our emergency laboratory has received a total of 55431 samples during the study period. Total test request forms received were 34644. Monthly distribution of total number of samples received, number of pre-analytical errors and pre-analytical error rate were shown in Table 2. Total number of pre-analytical errors were 1089 which accounted for $1.96 \%$ of total samples received. Distribution of number of pre-analytical errors from different ICUs and wards during the study period were shown in Table 3.

Haemolysed samples, clotted samples and samples with insufficient volume were contributed to $37 \%$, $26 \%$ and $15 \%$ of the total pre-analytical errors respectively.

Pre-analytical phase Quality indicators of emergency laboratory as per the IFCC WG-LEPS and performance levels were shown in Table 4.
There were 22 errors related to patient identification (Q I5) with $0.63 \%$ of obtained QI and six sigma values of 4.7. Errors concerning physician identification (QI 6) were 11 with $0.31 \%$ of obtained QI and six sigma values of 4.9. Errors concerning test input (QI 7) were 44 with $0.12 \%$ of obtained QI and six sigma values of 4.5. There were 22 samples lost-not received (QI 8) with $0.039 \%$ of obtained QI and six sigma values of 4.9. Samples collected in inappropriate container (QI 9) were 32 with $0.057 \%$ of obtained QI and six sigma values of 4.8 .

Haemolysed samples (QI 10) constituted 403 of total pre-analytical errors with $0.72 \%$ of obtained QI and six sigma values of 3.9. There were 284 clotted samples (QI 11) with $0.51 \%$ of obtained QI and six sigma values of 4.1. Samples with insufficient sample volume (QI 12) were 163 with $0.29 \%$ of obtained QI and six sigma values of 4.3. Samples with inadequate sample-anticoagulant volume ratio (QI 13) were 76 with $0.13 \%$ of obtained QI and six sigma values of 
4.5. Improperly labelled samples (QI 15) were 32 with $0.05 \%$ of obtained QI and six sigma values of 4.8. We have observed lowest six sigma value 3.9 for QI 10 and highest value 4.9 for QI 6 \& QI 8.

Table 2: Total Number of samples received, number of pre-analytical errors and percentage of Preanalytical errors during Jan 2019 to Dec 2019.

\begin{tabular}{|lccc|}
\hline Month & $\begin{array}{c}\text { Number } \\
\text { of samples } \\
\text { received }\end{array}$ & $\begin{array}{c}\text { Number } \\
\text { of pre- } \\
\text { analytical } \\
\text { errors }\end{array}$ & $\begin{array}{c}\text { Pre- } \\
\text { analytical } \\
\text { errors (\%) }\end{array}$ \\
\hline Jan-2019 & 4378 & 91 & 2.07 \\
Feb-2019 & 4632 & 98 & 2.11 \\
Mar-2019 & 4467 & 79 & 1.76 \\
Apr-2019 & 4298 & 105 & 2.44 \\
May-2019 & 4673 & 93 & 1.99 \\
Jun-2019 & 4574 & 87 & 1.90 \\
Jul-2019 & 4392 & 91 & 2.07 \\
Aug-2019 & 5623 & 86 & 1.52 \\
Sep-19 & 4537 & 84 & 1.85 \\
Oct-19 & 4552 & 85 & 1.86 \\
Nov-19 & 4629 & 99 & 2.13 \\
Dec-19 & 4676 & 91 & 1.94 \\
Total & 55431 & 1089 & 1.96 \\
\hline
\end{tabular}

\section{Discussion}

In the present study we have used harmonised QIs to assess pre-analytical phase of emergency laboratory. According to IFCC-WGLEPS, Quality indicator's with $25^{\text {th }}$ percentile value represents the best performance, $50^{\text {th }}$ percentile value represents the most common/frequent performance and $75^{\text {th }}$ percentile value represents the worst performance [11]. Most of our quality indicators showed optimal performance except QI 10 which in desirable range according to specifications laid by IFCC-WGLEPS [11].

Among all the quality indicators we have assessed QI 10 , haemolysed samples were $0.72 \%$ as compared to $0.4 \%$ by Grecu [12], $0.7 \%$ by Chawla et al [14] and $0.77 \%$, as reported by Lippi et al [15]. Our study reports $0.51 \%$ of clotted samples as compared to $0.77 \%$ by Grecu [12] and $0.25 \%$ by Lippi et al [15].

Our study reported six sigma metric value for haemolysed samples as 3.9 and clotted samples as 4.1, whereas Grecu et al [12] have reported 4.2 for haemolysed, 4.0 for clotted samples. Sciacovelli et al [11] has reported a six sigma values of 3.6 and 4.4 for Haemolysed and clotted samples respectively.

Sigma values for inadequate sample-anticoagulant volume ratio and samples with insufficient sample volume were 4.5 and 4.3 against values reported

Table 3: Distribution of number of pre-analytical errors from different ICUs and wards from Jan-2019 to Dec-2019.

\begin{tabular}{|lcccccccccccc|}
\hline Month & Casualty & ICU & SICU & NICU & MMWD & FMWD & MSWD & FSWD & PWD & OGWD & Chest WD & Total \\
\hline Jan-2019 & 17 & 12 & 6 & 8 & 7 & 5 & 9 & 3 & 8 & 10 & 6 & 91 \\
Feb-2019 & 26 & 14 & 10 & 9 & 6 & 9 & 4 & 5 & 6 & 7 & 2 & 98 \\
Mar-2019 & 18 & 8 & 8 & 10 & 12 & 4 & 3 & 4 & 4 & 5 & 3 & 79 \\
Apr-2019 & 23 & 11 & 14 & 12 & 8 & 7 & 7 & 3 & 7 & 11 & 2 & 105 \\
May-2019 & 14 & 17 & 8 & 14 & 10 & 5 & 5 & 2 & 10 & 5 & 3 & 93 \\
Jun-2019 & 23 & 9 & 12 & 11 & 7 & 3 & 8 & 3 & 4 & 6 & 1 & 87 \\
Jul-2019 & 23 & 16 & 13 & 9 & 5 & 4 & 3 & 5 & 6 & 5 & 2 & 91 \\
Aug-2019 & 17 & 15 & 9 & 13 & 6 & 3 & 6 & 2 & 5 & 7 & 3 & 86 \\
Sep-2019 & 16 & 12 & 7 & 8 & 8 & 7 & 5 & 2 & 8 & 9 & 2 & 84 \\
Oct-2019 & 24 & 9 & 8 & 9 & 11 & 2 & 3 & 7 & 4 & 6 & 2 & 85 \\
Nov-2019 & 18 & 11 & 12 & 15 & 8 & 4 & 5 & 4 & 9 & 9 & 4 & 99 \\
Dec-2019 & 21 & 8 & 13 & 12 & 10 & 2 & 7 & 3 & 5 & 7 & 3 \\
Total & 240 & 142 & 120 & 130 & 98 & 55 & 65 & 43 & 76 & 87 & 33 & 1089 \\
\hline
\end{tabular}

Abbreviations: MMWD=Male Medical Ward, FMWD=Female Medical ward, MSWD=male surgical ward, FSWD=Female surgical ward, PWD=Paediatric ward, OGWD=Obstetrics \& Gynaecology ward. 
by Grecu et.al [12] 5.6,4.8 and Sciacovelli et al [11] 5.0,3.9 respectively.

In the current study we have made efforts to use harmonised QIs to monitor and quantify the performance of several steps of pre-analytical phase of our laboratory. These QIs are comparable with Quality specifications laid by IFCC-WGLEPS [11].
The present study showed most common preanalytical errors are related to haemolysed samples followed by inadequate sample-anticoagulant volume ratio and samples with insufficient sample volume. None of our QIs showed unacceptable performances according to six sigma metrics in our study.

Table 4: Assessment of pre-analytical phase quality indicators of emergency laboratory as per the IFCC WG-LEPS and six sigma.

\begin{tabular}{|c|c|c|c|c|c|c|}
\hline QI code & Description & $\begin{array}{l}\text { No of } \\
\text { errors }\end{array}$ & $\begin{array}{c}\text { QI obtained } \\
\text { value as per } \\
\text { description (\%) }\end{array}$ & $\begin{array}{l}\text { IFCC-based } \\
\text { performance } \\
\text { level [11] }\end{array}$ & $D P M$ & $\begin{array}{l}\text { Sigma } \\
\text { value }\end{array}$ \\
\hline QI 5: Identification & $\begin{array}{l}\text { Number of requests with errors concerning } \\
\text { patient identification / Total number of } \\
\text { requests }\end{array}$ & 22 & 0.063 & Optimal & 635 & 4.7 \\
\hline QI 6: Identification & $\begin{array}{l}\text { Number of requests with errors concerning } \\
\text { physician identification / Total number of } \\
\text { requests }\end{array}$ & 11 & 0.03 & Optimal & 317 & 4.9 \\
\hline QI 7: Test request & $\begin{array}{l}\text { Number of requests with errors concerning } \\
\text { test input / Total number of requests }\end{array}$ & 44 & 0.127 & Optimal & 1270 & 4.5 \\
\hline QI 8: Samples & $\begin{array}{l}\text { Number of samples lost-not received / Total } \\
\text { number of samples }\end{array}$ & 22 & 0.039 & Optimal & 397 & 4.9 \\
\hline QI 9: Samples & $\begin{array}{l}\text { Number of samples collected in } \\
\text { inappropriate container / Total number of } \\
\text { samples }\end{array}$ & 32 & 0.057 & Optimal & 577 & 4.8 \\
\hline QI 10: Samples & $\begin{array}{l}\text { Number of haemolysed samples / Total } \\
\text { number of samples }\end{array}$ & 403 & 0.72 & Desirable & 7270 & 3.9 \\
\hline QI 11: Samples & $\begin{array}{l}\text { Number of clotted samples / Total number of } \\
\text { samples with anticoagulant }\end{array}$ & 284 & 0.51 & Optimal & 5123 & 4.1 \\
\hline QI 12: Samples & $\begin{array}{l}\text { Number of samples with insufficient sample } \\
\text { volume / Total number of samples }\end{array}$ & 163 & 0.29 & Optimal & 2940 & 4.3 \\
\hline QI 13: Samples & $\begin{array}{l}\text { Number of samples with inadequate sample- } \\
\text { anticoagulant volume ratio / Total number of } \\
\text { samples with anticoagulant }\end{array}$ & 76 & 0.137 & Optimal & 1371 & 4.5 \\
\hline QI 15: Samples & $\begin{array}{l}\text { Number of improperly labelled samples / } \\
\text { Total number of samples }\end{array}$ & 32 & 0.057 & Optimal & 577 & 4.8 \\
\hline
\end{tabular}

\section{Limitations}

We have examined the QIs which are more suitable to our quality program, however the present study did not examine QI 1-4, QI 14 and QI 16. Identifying and recording of all QIs require lots of time efforts by the laboratory staff which may not be feasible during night shifts in emergency laboratory.

\section{Conclusion}

The present study showed haemolysed samples followed by inadequate sample-anticoagulant volume ratio and samples with insufficient sample volume were most common pre-analytical phase errors. Pre-analytical phase performance of our emergency laboratory complies with the quality specifications laid by IFCC-WGLEPS. Implementation of harmonised QIs assures the comparability of laboratory findings with different laboratories across the world. Harmonised QIs also play a key role in ensuring the targeted continuous improvement activities aiming to reduce the risk of errors. 


\section{Acknowledgement}

Laboratory technicians involved in data collection, ESIC Medical College \& PGIMSR, KK Nagar, Chennai, Tamil Nadu, India.

\section{Conflicts of interest}

Authors declare no conflicts of interest.

\section{References}

[1] Plebani M. Quality indicators to detect pre-analytical errors in laboratory testing. Clin Biochem Rev. 2012; 33(3):85-88.

[2] Da Rin G. Pre-analytical workstations: a tool for reducing laboratory errors. ClinChim Acta. 2009; 404(1):68-74.

[3] Guder WG. History of the pre analytical phase: a personal view. Biochem Med. 2014; 24(1):25-30.

[4] Lippi G, Chance JJ, Church S, Dazzi P, Fontana R, et al. Preanalytical quality improvement: from dream to reality. Clin Chem Lab Med. 2011; 49(7):1113-1126.

[5] Plebani M, Carraro P. Mistakes in a stat laboratory: types and frequency. Clin Chem. 1997; 43(8 Pt 1):1348-1351.

[6] Karcher DS, Lehman CM. Clinical consequences of specimen rejection: a college of american pathologists Q-probes analysis of 78 clinical laboratories. Arch Pathol Lab Med. 2014; 138(8):1003-1008.

[7] Barth JH. Clinical quality indicators in laboratory medicine. Ann ClinBiochem 2012; 49(Pt 1):9-16.

[8] Plebani M. The detection and prevention of errors in laboratory medicine. Ann Clin Biochem. 2010; 47(Pt 2):101110.

[9] ISO 15189: 2012. Medical laboratories - requirements for quality and competence, 2012.

[10] West J, Atherton J, Costelloe SJ, Pourmahram G, Stretton A, et al. Preanalytical errors in medical laboratories: a review of the available methodologies of data collection and analysis. Ann Clin Biochem. 2017; 54(1):14-19.

[11] Sciacovelli L, Lippi G, Sumarac Z, West J, Castro IGDP, et al. Quality Indicators in Laboratory Medicine: the status of the progress of IFCC Working Group "Laboratory Errors and Patient Safety" project. Clin Chem Lab Med. 2017; 55(3):348-357.

[12] Grecu DS, Vlad DC, Dumitrascu V. Quality indicators in the preanalytical phase of testing in a stat laboratory. Lab Med. 2014; 45(1):74-81.

[13] Westgard JO, Westgard SA. The quality of laboratory testing today. An assessment of $\sigma$ metrics for analytic quality using performance data from proficiency testing surveys and the CLIA criteria for acceptable performance. Am J Clin Pathol. 2006; 125(3):343-354.

[14] Chawla R, Goswami B, Singh B, Chawla A, Gupta VK, et al. Evaluating laboratory performance with quality indicators. Lab Med. 2010; 41(5):297-300.

[15] Lippi G, Bassi A, Brocco G, Montagnana M, Salvagno GL, et al. Preanalytic error tracking in a laboratory medicine department: results of a 1-year experience. Clin Chem. 2006; 52(7):1442-1443. 